\title{
Evaluation of renoprotective effect of calcium channel blockers in coronary angiography patients
}

Burçin Meryem Atak Tel · Memnune Sena Ulu · Sinan Kazan · Mehmet Polat

Department of Internal Medicine, Afyon Kocatepe University, Faculty of Medicine, Afyon, Turkey

\section{ABSTRACT}

Aim: To evaluate the effectiveness of contrast-induced acute kidney injury (CI-AKI) prophylaxis retrospectively, using calcium channel blockers (CCB) before and after contrast exposure and comparing them with patients using angiotensin converting enzyme inhibitors (ACEI), which has not been explored by many studies.

Methods: The study was performed in Afyon Kocatepe University, Faculty of Medicine Research Hospital, Cardiology Department between January 2014 and June 2016. Eighty patients using dihydropyridine (amlodipine $10 \mathrm{mg}$ ), non-dihydropyridine (diltiazem $60 \mathrm{mg}$ ) CCB or ACEI in the form of monotherapy before coronary angiography were included.

Results: In the CCB and ACEI group, CI-AKI development rates were $15.7 \%(\mathrm{n}=8)$ and $24.1 \%(\mathrm{n}=7)$, respectively ( $p=0.383$; Fisher's exact test). When the CCB group was evaluated as dihydropyridine and non-dihydropyridine subsets, CI-AKI development rates were found to be similar as well $(p=$ 0.445; Fisher's exact test) in each subset.

Conclusion: In our study, we evaluated one of today's important dilemma; the methods related to the prophylaxis of CI-AKI. Our study shows that there is no difference in the development of CI-AKI between patients using the calcium channel blocker group drugs and ACEI as monotherapy. However, in our study, the mean age of patients using CCB was significantly higher than the group using ACEI. Keywords: Contrast-induced acute kidney injury, calcium channel blockers, angiotensin converting enzyme inhibitors, renoprotective effect.

(C) 2020 experimentalbiomedicalresearch.com

$\triangle$ Dr. Burçin Meryem Atak Tel

Department of Internal Medicine, Afyon Kocatepe

University, Faculty of Medicine, Afyon, Turkey

E mail: burcinatak@hotmail.com

Received: 2020-04-19/Revisions: 2020-04-28

Accepted: 2020-05-06 / Publication Date: 2020-06-13

\section{Introduction}

Contrast media (CM) has a plethora of applications in routine non-invasive or percutaneous invasive imaging examinations and therapeutic interventions. Unfortunately, the use of $\mathrm{CM}$ is associated with a number of complications, the most serious being contrastinduced acute kidney injury (CI-AKI) [1].

A general definition of CI-AKI is an impairment in renal function occurring within 3 days following the intravascular administration of $\mathrm{CM}$ and the absence of an alternative etiology [2]. Contrast media administration has been said to be the third leading cause of 
hospital-acquired acute renal failure in the past 3 decades [3]. Chronic kidney disease, dehydration, diabetes mellitus (DM), advanced age, increased volume of $\mathrm{CM}$ and recurrent administrations are well-known risk factors of CI-AKI [4].

In spite of the vast clinical importance of CIAKI, its understanding and the pathophysiology behind CI-AKI is not fully explained [2, 3]. Most reviews show a complex pathophysiology overlaying medullary ischemia and hypoxia, oxidant damage, intratubular obstruction, hypertonicity, plasma viscosity and many pathways including endothelins, nitric oxide, reactive oxygen species, prostaglandins and adenosine [5].

Calcium has been proposed as a mediator of the vasoconstrictor response to $\mathrm{CM}$ [6]. Also Intracellular $\mathrm{Ca}^{2+}$ overload is considered to be a key factor in CI-AKI [3]. The rationale is based on the fact that while in normal subjects, the $\mathrm{Na}^{+}-\mathrm{Ca}^{2+}$ exchanger pumps $\mathrm{Ca}^{2+}$ outside the renal tubular epithelial cells to keep intracellular $\mathrm{Ca}^{2+}$ low. Under the effect of $\mathrm{CM}$, the $\mathrm{Na}^{+}-\mathrm{Ca}^{2+}$ exchanger can reversibly extrude $\mathrm{Na}^{+}$for $\mathrm{Ca}^{2+}$ influx, thereby leading to intracellular $\mathrm{Ca}^{2+}$ overload, which is considered a key factor in ischemic cell injury and in CIAKI [7]. The increase in intracellular calcium provokes a vasoconstrictive response in intrarenal circulation and would been important mediator of epithelial cell apoptosis and necrosis. Thus, calcium channel blockers (CCB) have been hypothesized to have protective effects against CI-AKI [8]. The CCB attenuated the vasoconstrictor response of $\mathrm{CM}$ in animal studies, although prophylactic use of CCB has not gained wide acceptance [6]. Literature show opposing results; some authors suggesting them to be protective $[9,10]$, others finding no benefit at all [11-13]. However, these researches are very old and more recent studies are needed. In this study we compared patients who used dihydropyridine (amlodipine $10 \mathrm{mg}$ ), nondihydropyridine (diltiazem $60 \mathrm{mg}$ ) CCB and ACEI; and underwent coronary angiography. We compared serum creatinine, blood urea nitrogen (BUN), urea levels and glomerular filtration rates (GFR) before and 315 days after the coronary angiography. We planned to examine the changes in the GFR values by taking demographic data into consideration.

\section{Materials and methods}

We used a definition of CI-AKI which is widely accepted; impairment in renal function occurring within 3 days following the intravascular administration of $\mathrm{CM}$ and the absence of an alternative etiology [2].

The study included 80 patients using dihydropyridine, non-dihydropyridine CCB and ACEI from 4027 patients who underwent coronary angiography in the Cardiology department of Afyon Kocatepe University, Faculty of Medicine, Research Hospital between January 2014 and June 2016. Information about patients was obtained by retrospectively examining patient files. Prior to the study, the necessary ethics committee approval was obtained (Decision no; 29-52016). All procedures performed in this study were in accordance with the ethical standards of the institutional and/or national research committee and with the 1964 Helsinki declaration and its later amendments or comparable ethical standards.

Inclusion and exclusion criteria: Of the 4027 patients; only 80 patients used dihydropyridine (amlodipine 10mg), nondihydropyridine (diltiazem 60mg) or ACEI before coronary angiography as monotherapy. 2635 patients using multidrug therapies were excluded. Also 
1074 patients were excluded because they were not using CCB or ACEI.

Individuals under the age of 18 and over 85 were not included in the study (total of 84 patients). Data was obtained by retrospectively scanning patient files. Patients were included in the study if the registration data were sufficient. Patients were excluded if serum creatinine, BUN, urea levels and GFR value within 3 to 15 days of coronary angiography were not in the database. Also patients with missing demographic data were excluded. 154 patients were excluded because of a missing data in their files.

\section{Statistical Analysis}

The data of the patients' who are included in the study has been collected and submitted in a database for the study. These variables include; age, gender, smoking habits, body mass index (BMI), hypertension duration (in years), systolic and diastolic blood pressure measurements, comorbid disease, patients laboratory findings (before and after contrast exposure) including hemogram, BUN, serum creatinine, urea, glomerular filtration rate (GFR), lipid analysis, and electrolytes. Data was analyzed using IBM SPSS 18.0. Descriptive statistical results of the study data were expressed as arithmetic mean \pm standard deviation. While evaluating the groups, patient distributions were given as frequencies. The data obtained were evaluated primarily with descriptive statistics. The suitability of the data to the parametric conditions was evaluated with the Kolmogorov-Smirnov test. Mann-Whitney $\mathrm{U}$ test was used for the quantitative evaluation between the CCB and ACEI groups. Wilcoxon Test was used to compare the median values of the two dependent groups, and the Chi Square test was used to compare the categorical data and the groups. In the results obtained from the statistical tests applied, it was considered significant when a $95 \%$ confidence interval (CI) and the p-value below 0.05 .

\section{Results}

When the demographic data of the patients included in the study were evaluated, 55 $(68.8 \%)$ were male and $25(31.2 \%)$ were female. There were 29 (56\%) men, 22 (44\%) women in the patient group using calcium channel blocker; and $26(89 \%)$ men and 3 (11\%) women in the ACEI group. There was a significant difference in gender distribution between the two groups ( $p=0.002$ ). The mean age of the patients was $60.4 \pm 12.5$. When analyzed as CCB and ACEI groups, the average age of patients using $\mathrm{CCB}$ was $62.6 \pm 12.6$, and the average age of the patient group using ACEI was $56.5 \pm 11.5$. The mean ages of the two groups were found to be statistically different $(p=0.01)$. BMI was similar between the CCB group and the ACEI group ( $p=0.222$ ). When systolic blood pressures of patients were evaluated, mean systolic blood pressure was $132.9 \pm 15.3$ (range: $110-180$ ) $\mathrm{mmHg}$ in the CCB group and $119.1 \pm 10.6$ (range: 86-140) $\mathrm{mmHg}$ in the group using ACEI. Systolic blood pressure was significantly higher in the $\mathrm{CCB}$ group than the ACEI group ( $p<0.001$ ). Again, when groups using $\mathrm{CCB}$ and ACEI were compared, diastolic blood pressures were similar $(p=0.663)$. The duration of involved drug use in both groups was similar $(p<0.233)$. Of the 80 patients who are enrolled in the study, 25 had no other comorbid disease. $31(38,8 \%)$ had DM, $5(6.3 \%)$ had a history of cerebrovascular disease, $7 \quad(8.8 \%)$ had congestive heart failure, $3(3.8 \%)$ had peripheral arterial disease, $15(18.8 \%)$ had hyperlipidemia, 10 (12.5\%) had chronic lung disease. Disease distributions were similar in CCB and ACEI groups ( $p>0.5)$. 
When the drugs used by patients were classified, 21 people were using nondihydropyridine (diltiazem 60mg), 30 people were using dihydropyridine (amlodipine $10 \mathrm{mg}$ ) and 29 people were using ACEI.

When staged according to the JNC8 report, only 10 of the 80 patients were found to have optimal systolic and diastolic blood pressure. Despite the use of $\mathrm{CCB}$, blood pressure levels of 5 patients were found to be stage- 2 hypertension. It was found that patients using trandolapril had more effective control over blood pressure compared to patients using CCB.
The laboratory values of the two groups are presented in Table 1. When the laboratory values of the two groups were compared, the hemoglobin, hematocrit, platelet, fasting blood glucose, uric acid, calcium (Ca), AST, ALT, lipid panel (LDL, VLDL, HDL, total cholesterol, triglyceride) values were also similar between the CCB group and the ACEI group. There was a significant difference between phosphorus and HbA1c levels between the two groups. Serum phosphorus level in the CCB group was 2.6-4.5 (mean $3.51 \pm 0.5) \mathrm{mg} /$ $\mathrm{dl}$, and in the group using ACEI 1.5-4.2 (mean $3.1 \pm 0.6) \mathrm{mg} / \mathrm{dl}(p=0.043)$. The HbA1c level

Table 1. Comparison of laboratory parameters of two groups.

\begin{tabular}{|l|c|c|c|c|c|}
\hline \multirow{2}{*}{$\begin{array}{l}\text { Laboratory } \\
\text { Parameters }\end{array}$} & \multicolumn{2}{|c|}{ CCB Group } & \multicolumn{2}{c|}{ ACEI Group } & \multirow{2}{*}{ p-value } \\
\cline { 2 - 5 } & Min-max & Mean & Min-max & Mean & \\
\hline Hb(g/dl) & $9-17$ & $12.7 \pm 2.0$ & $9,6-17,0$ & $13.07 \pm 2.1$ & 0.538 \\
\hline Htc (\%) & $28-57$ & $40.3 \pm 6.5$ & $29-50$ & $40.6 \pm 6.1$ & 0.641 \\
\hline Plt (x10 $)$ & $66-369$ & $232.3 \pm 64.3$ & $177-352$ & $239.4 \pm 55$ & 0.944 \\
\hline FBG (mg/dl) & $60-377$ & $142.6 \pm 65,9$ & $74-358$ & $158.5 \pm 76.5$ & 0.597 \\
\hline Uric Acid (mg/dl) & $2,8-27$ & $5.8 \pm 3.5$ & $2.8-12$ & $5.6 \pm 2$ & 0.768 \\
\hline Ca ${ }^{++}$(mg/dl) & $7,9-10$ & $9.1 \pm 8.7$ & $7,8-10$ & $9.1 \pm 0.5$ & 0.864 \\
\hline Phosphorus (mg/dl) & $2,6-4,5$ & $3.51 \pm 0.5$ & $1,5-4,2$ & $3.1 \pm 0.6$ & 0.043 \\
\hline K+ (mEq/l) & $3,1-5,5$ & $4.4 \pm 0.5$ & $3,2-5,1$ & $4.4 \pm 0.4$ & 0.028 \\
\hline HbA1c \% & $5,3-12$ & $7.8 \pm 1.8$ & $9,0-13,0$ & $10.4 \pm 1.6$ & $\mathbf{0 . 0 1 7}$ \\
\hline AST (U/L) & $13-78$ & $26.7 \pm 12.5$ & $12-206$ & $40.4 \pm 38.7$ & 0.222 \\
\hline ALT (U/L) & $5-167$ & $28.8 \pm 31.4$ & $8-61$ & $22.8 \pm 12.08$ & 0.980 \\
\hline LDL (mg/dl) & $25-228$ & $114.2 \pm 46.3$ & $51-212$ & $108.5 \pm 40.9$ & 0.572 \\
\hline VLDL (mg/dl) & $9-106$ & $31.8 \pm 17.5$ & $9-63$ & $29.2 \pm 14.2$ & 0.663 \\
\hline HDL (mg/dl) & $14-68$ & $37.7 \pm 12.4$ & $16-61$ & $36.4 \pm 10.1$ & 0.676 \\
\hline $\begin{array}{l}\text { Total } \\
\text { cholesterol(mg/dl) }\end{array}$ & $120-324$ & $168.1 \pm 58.8$ & $84-284$ & $161.1 \pm 48.6$ & 0.420 \\
\hline Triglyceride(mg/dl) & $85-531$ & $151.4 \pm 94.0$ & $48-316$ & $147.5 \pm 72.3$ & 0.890 \\
\hline
\end{tabular}

CCB: calcium channel blockers, ACEI: angiotensin converting enzyme inhibitors Hb: Hemoglobin, Htc: hematocrit, Plt: Platelet. FBG: fasting blood glucose. 
was $5.3-12 \%$ (mean: $7.8 \pm 1.8$ ) in the $\mathrm{CCB}$ group and $9-13 \%(10.4 \pm 1.6)$ in the group using ACEI $(p=0.017)$. Serum potassium level was $4.2 \pm 0.5 \mathrm{mEq} / \mathrm{dl}$ in the CCB group, and $4.4 \pm$ $0.4 \mathrm{mEq} / \mathrm{dl}$ in the ACEI group. There was a significant difference between the potassium levels of two groups $(p=0.02)$.

Pre-contrast exposure serum creatinine, BUN, urea and GFR were similar ( $p$ values $=0.359$, $0.904,0.707,0.426$, respectively). The mean urea of the group using CCB after contrast exposure was $43.1 \pm 23.0 \mathrm{mg} / \mathrm{dl}$, and $38.9 \pm$ $23.6 \mathrm{mg} / \mathrm{dl}$ in the ACEI group $(p=0.08) . \mathrm{Cr}$ value of CCB group was $1.06 \pm 1.4 \mathrm{mg} / \mathrm{dl}$, and $1.0 \pm 0.4 \mathrm{mg} / \mathrm{dl}$ in the ACEI group $(p=0.11)$. After contrast exposure, GFR was calculated as $85.9 \pm 27.9(\mathrm{ml} / \mathrm{min} / 1.73 \mathrm{~m} 2)$ for the CCB group and $88.9 \pm 33.9(\mathrm{ml} / \mathrm{min} / 1.73 \mathrm{~m} 2)$ for the ACEI group. When the two groups were compared in terms of GFR levels after contrast exposure, they were found similar $(p=0.818)$.

In the $\mathrm{CCB}$ and ACEI groups, CI-AKI development rates were $15.7 \% \quad(n=8)$ and $24.1 \%(n=7)$, respectively. The two groups were similar in terms of CI-AKI development rates ( $p=0.383$; Fisher's exact test).

When the CCB group was evaluated as dihydropyridine and nondihydropyridine groups, the rates of CI-AKI were similar $(p=$ 0.445; Fisher's exact test).

\section{Discussion}

CI-AKI has become an important problem as a result of the increased use of contrast today. Again, it increases the life-threatening complications such as sepsis, bleeding, and respiratory failure, and increases the hospital stay and leads to an increase in medical costs. Prevention and treatment of such an important complication is very essential for the physician, patient and the country's economy. The data obtained up to this day confirms the idea that the volume expansion method is the most important method for reducing the risk of CIAKI [14]. But the pathophysiology behind CIAKI is not fully explained $[2,3]$. Therefore search for a prophylaxis of CI-AKI still continues.

Intracellular $\mathrm{Ca}^{2+}$ overload is an important factor in ischemic cell injury and considered to be a key factor in CI-AKI pathophysiology [3]. Therefore $\mathrm{CCB}$, which could prevent intracellular $\mathrm{Ca}^{2+}$ overload, have been suggested as a protective measure to prevent CI-AKI [7]. However previous data shows conflicting results. Most of the animal studies on rats showed promising results. Yu-Yan Fan et al., Aritomi et al. and Duan et al. all showed similar renoprotective effects of CCB [15-17]. Beyazal et al. [14] compared isotonic sodium chloride infusion alone, 5\% dextrose solution with sodium bicarbonate infusion and isotonic sodium chloride infusion plus 3 days of CCB therapy (one day before and two days after the contrast exposure) for CI-AKI prophylaxis. They find no significant difference between groups.

Arici et al. [18] also find no significant difference in a prospective study with patients pretreated with amlodipine; a dihydropyridine CCB; than placebo. Whereas Russo et al. [10] reported that $\mathrm{CCB}$ nifedipine may prevent $\mathrm{AKI}$ induced by hyperosmolar contrast agent. Neumayer et al. [9] investigated a total of 35 patients after intravascular administration of contrast media to determine the effects on renal function of a 3-day treatment with the CCB nitrendipine $(n=16)$, compared the findings in a placebo-treated control group $(n=19)$. Prophylactic use of nitrendipine preserved the glomerular filtration rate, whereas control patients showed a significant $(27 \%)$ reduction in GFR two days after contrast-media injection $(p \leq 0.01)$. As a result, it was emphasized that 
nitrendipine, a nondihydropyridine $\mathrm{CCB}$, could decrease the risk of CI-AKI [9].

In our study we compared two groups, who were using $\mathrm{ACEI}$ and $\mathrm{CCB}$ as monotherapy; and found no significant difference in development of CI-AKI. But this can be an effect of ACEI as well as CCB. ACEI also have been used to prevent CI-AKI in the past. Gupta et al. conducted a study of 71 patients who underwent coronary angiography and concluded that ACEI is effective against CIAKI compared to placebo [19]. But more recent studies find no beneficial effect of ACEI. Furthermore Toprak et al. reported that in a randomized controlled study of 80 patients included; five patients $(8.3 \%)$ in the ACEI group and 1 patient (3\%) in control group developed CI-AKI and this difference was statistically significant $(p=0.02)$. They concluded that using ACEI is a risk factor for development of CI-AKI [20]. In our study, 8 (15.7\%) patients in CCB group and 7 (24.1\%) patients in ACEI group developed CI-AKI. The two groups were similar in terms of CI-AKI development rates statistically $(p=0.383)$. This may be the result of both drugs lowering the risk factor of CI-AKI equally.

Age is a direct risk factor of developing CIAKI. Especially elderly patients older than 7075 years are at risk of developing CI-AKI [7]. Hui et al. showed in their study that amlodipine, a $\mathrm{CCB}$, may decrease the risk of developing CMN in elderly patients [21]. In our study, the mean age of the CCB group was significantly higher than the ACEI group. The mean age of the patients in $\mathrm{CCB}$ group was $62.6 \pm 12.6$, against the mean age of the patients in ACEI group was $56.5 \pm 11.5(p=0.01)$. This could mean that $\mathrm{CCB}$ reduced the risk of older patients in the CCB group to a younger age risk level. Therefore two groups statistically appear indifferent in CI-AKI development rates.
Oguzhan et al. compared hydration therapy alone, versus valsartan-amlodipine combination plus hydration treatment in patients who have stage-II chronic kidney disease and going through coronary angiography. CI-AKI rates was $17.8 \%(\mathrm{n}=8)$ in the CCB/ARB plus hydration group and $6.7 \%$ $(n=3)$ in the only hydration group. As a result, they showed that amlodipine and valsartan treatment did not decrease the risk of CI-AKI [22]. Davidson et al. prospectively examined 1144 patients undergoing cardiac catheterization. They showed that the risk of developing CI-AKI does not decrease in patients using various CCB drugs [23]. In their prospective randomized study, Arıc1 et al. [18] divided the 29 patients into two groups of amlodipine $(n=15)$ and placebo $(n=14)$. Only one patient developed CI-AKI in each group. Two groups were similar statistically regarding CI-AKI development rates (amlodipine group: $6.6 \% \mathrm{n}=1$; placebo group: $7.1 \% \mathrm{n}=1$ ). Although our study was designed retrospectively, it is important to show similar results and rates with these three prospective studies.

The main limitations of our study are retrospective study design and relatively small sample size. Also; age difference between two groups and the fact that we could not randomize groups for contrast dose and their hydration status is a limitation of our study.

\section{Conclusion}

In our study, we evaluated one of today's important dilemma; the methods related to the prophylaxis of CI-AKI. Our study shows that there is no difference in the development of CIAKI between patients using the CCB and ACEI as monotherapy. However, in our study, the mean age of patients using CCB was significantly higher than the group using ACEI. The volume expansion method is the most 
favorable method for reducing the risk of CI$\mathrm{AKI}$ and the pathophysiology behind CI-AKI is not fully explained. Therefore we need more studies exploring CI-AKI prophylaxis.

Funding: There is no financial support and sponsorship

Conflict of Interest: The authors declare that they have no conflict of interest.

Ethical statement: The study was reviewed and approved by the local ethics committee (Decision no; 29-5-2016)

\section{ORCID iD of the author(s)}

Burçin MA Atak Tel / 0000-0003-4201-9757

Memnune Sena Ulu / 0000-0003-0085-2193

Sinan Kazan / 0000-0001-7290-4680

Mehmet Polat / 0000-0003-1648-5543

\section{References}

[1]Vergadis C, Festas G, Spathi E, et al. Methods for Reducing Contrast Use and Avoiding Acute Kidney Injury During Endovascular Procedures. Curr Pharm Des. 2019; 25(44): 4648-55.

[2]Persson PB, Tepel M. Contrast mediuminduced nephropathy: the pathophysiology. Kidney Int Suppl. 2006;(100): S8-10.

[3]Yang D, Yang D, Jia R, et al. $\mathrm{Na}+/ \mathrm{Ca} 2+$ exchange inhibitor, KB-R7943, attenuates contrast-induced acute kidney injury. J Nephrol. 2013;26(5): 877-85.

[4]Zhao K, Gao Q, Zong C, et al. Cordyceps sinensis prevents contrast-induced nephropathy in diabetic rats: its underlying mechanism. Int J Clin Exp Pathol. 2018; 11(12): 5571-80.

[5]Hiremath S, Velez JCQ. Preventing a nonexistent entity: the curious case of contrast and acute kidney injury. Curr Opin Nephrol Hypertens. 2020; 29(1): 152-60.
[6]Briguori C, Tavano D, Colombo A. Contrast agent--associated nephrotoxicity. Prog Cardiovasc Dis. 2003; 45(6): 493-503.

[7]Andreucci M, Faga T, Serra R, et al. Update on the renal toxicity of iodinated contrast drugs used in clinical medicine. Drug Healthc Patient Saf. 2017; 9: 25-37.

[8]Andreucci M, Faga T, Pisani A, et al. Prevention of contrast-induced nephropathy through a knowledge of its pathogenesis and risk factors. ScientificWorldJournal. 2014; 2014: 823169.

[9]Neumayer HH, Junge W, Kufner A, et al. Prevention of radiocontrast-media-induced nephrotoxicity by the calcium channel blocker nitrendipine: a prospective randomised clinical trial. Nephrol Dial Transplant. 1989; 4(12): 1030-36.

[10]Russo D, Testa A, Della Volpe L, et al. Randomised prospective study on renal effects of two different contrast media in humans: protective role of a calcium channel blocker. Nephron. 1990; 55(3): 254-57.

[11]Khoury Z, Schlicht JR, Como J, et al. The effect of prophylactic nifedipine on renal function in patients administered contrast media. Pharmacotherapy. 1995; 15(1): 5965.

[12]Solomon R, Werner C, Mann D, et al. Effects of saline, mannitol, and furosemide on acute decreases in renal function induced by radiocontrast agents. N Engl J Med. 1994; 331(21): 1416-20.

[13]Spangberg-Viklund B, Berglund J, Nikonoff $\mathrm{T}$, et al. Does prophylactic treatment with felodipine, a calcium antagonist, prevent low-osmolar contrast-induced renal dysfunction in hydrated diabetic and nondiabetic patients with normal or moderately reduced renal function? Scand J Urol Nephrol. 1996; 30(1): 63-68. 
[14]Beyazal H, Caliskan Z, Utac C. Comparison of effects of isotonic sodium chloride with diltiazem in prevention of contrast-induced nephropathy. Ren Fail. 2014; 36(3): 351-55.

[15]Aritomi S, Harada E, Sugino K, et al. Comparison of the cardioprotective and renoprotective effects of the L/N-type calcium channel blocker, cilnidipine, in adriamycin-treated spontaneouslyhypertensive rats. Clin Exp Pharmacol Physiol. 2015; 42(4): 344-52.

[16]Duan SB, Liu FY, Luo JA, et al. Nephrotoxicity of high- and low-osmolar contrast media. The protective role of amlodipine in a rat model. Acta Radiol. 2000; 41(5): 503-7.

[17]Fan YY, Kohno M, Nakano D, et al. Cilnidipine suppresses podocyte injury and proteinuria in metabolic syndrome rats: possible involvement of N-type calcium channel in podocyte. J Hypertens. 2010; 28(5): 1034-43.

[18]Arici M, Usalan C, Altun B, et al. Radiocontrast-induced nephrotoxicity and urinary alpha-glutathione S-transferase levels: effect of amlodipine administration. Int Urol Nephrol. 2003; 35(2): 255-61.

[19]Gupta RK, Kapoor A, Tewari S, et al. Captopril for prevention of contrast-induced nephropathy in diabetic patients: a randomised study. Indian Heart J. 1999; 51(5): 521-26.

[20]Toprak O, Cirit M, Bayata S, et al. The effect of pre-procedural captopril on contrastinduced nephropathy in patients who underwent coronary angiography. Anadolu Kardiyol Derg. 2003; 3(2): 98-103.

[21]Hui H, Li K, Li Z, et al. Protective effect of amlodipine against contrast agent-induced renal injury in elderly patients with coronary heart disease. Nan Fang Yi Ke Da Xue Xue Bao. 2012; 32(11): 1580-83.
[22]Oguzhan N, Cilan H, Sipahioglu M, et al. The lack of benefit of a combination of an angiotensin receptor blocker and calcium channel blocker on contrast-induced nephropathy in patients with chronic kidney disease. Ren Fail. 2013; 35(4): 434-39.

[23]Davidson CJ , Hlatky M, Morris KG, et al. Cardiovascular and renal toxicity of a nonionic radiographic contrast agent after cardiac catheterization. A prospective trial. Ann Intern Med. 1989; 110(2): 119-24. 